Références bibliographiques du dossier "L'enseignement supérieur, une compétition mondiale?»

Bernadette Plumelle

\title{
OpenEdition
}

Journals

Édition électronique

URL : http://journals.openedition.org/ries/273

DOI : $10.4000 /$ ries.273

ISSN : 2261-4265

Éditeur

Centre international d'études pédagogiques

Édition imprimée

Date de publication : 1 septembre 2007

Pagination : 145-159

ISBN : 978-2-85420-570-1

ISSN : $1254-4590$

Référence électronique

Bernadette Plumelle, «Références bibliographiques du dossier « L'enseignement supérieur, une compétition mondiale? » », Revue internationale d'éducation de Sèvres [En ligne], 45 | septembre 2007, mis en ligne le 23 juin 2011, consulté le 01 mai 2019. URL : http://journals.openedition.org/ries/273 DOI : 10.4000/ries. 273 


\section{Références bibliographiques}

\section{Bernadette Plumelle}

Les politiques de l'enseignement supérieur menées par les pays du Nord comme du Sud cherchent à répondre à de nombreuses missions dans un contexte de mondialisation et de forte compétition.

Avec des documents postérieurs à l'an 2000, cette bibliographie ${ }^{1}$, non exhaustive, a pour objectif de rendre compte des évolutions de ce secteur à l'étranger comme en France. Après des études comparatives sur l'enseignement supérieur, une première partie recense des documents sur les défis actuels de l'université : internationalisation des enseignements, mouvement de privatisation de ce secteur de l'éducation, importance accrue de la recherche et mise en place de démarches de qualité. Quelques études régionales et nationales complètent cette partie. La deuxième partie porte sur l'Europe et sur le processus de Bologne (1999), suivie de monographies sur des pays européens. Enfin, la dernière partie recense les rapports officiels sur l'enseignement supérieur en France ainsi que des publications sur les mutations de l'université française.

La majorité des documents mentionnés sont consultables au centre de ressources du Centre international d'études pédagogiques ou sont en ligne sur Internet.

Bibliographie arrêtée le 4 juillet 2007.

\section{DONNÉES COMPARATIVES SUR L'ENSEIGNEMENT SUPÉRIEUR}

OCDE: Organisation de coopération et de développement économiques/Paris, Regards sur l'éducation : les indicateurs de l'OCDE 2006, OCDE/Paris, 2006, 475 p., annexes

Ce rapport comprend des données tendancielles sur les diplômes avec des projections pour l'année 2014; des données sur les taux de poursuite dans l'enseignement supérieur et un aperçu de la mobilité des étudiants et de l'ampleur de l'internationalisation dans l'enseignement supérieur.

OCDE : Organisation de coopération et de développement économiques, Analyse des politiques d'éducation 2005-2006 : regards sur l'enseignement supérieur, OCDE/Paris, 2006, 180 p.

Cette édition d'Analyse des politiques d'éducation porte sur l'amélioration de la qualité, sur l'équité et l'efficience de l'enseignement supérieur. Elle reprend les documents se rapportant à la réunion des ministres de l'éducation de l'OCDE (Athènes, 27-28 juin 2006) et fournit une analyse des orientations concernant le marché international en expansion de l'enseignement supérieur.

1. Une bibliographie générale sur l'enseignement supérieur a été produite par l'INRP (voir «L'enseignement supérieur sous le regard des chercheurs » d’Olivier Rey, dans cet article); elle est complète et recense des centaines de références sur le sujet. Par ailleurs, l'auteur fournit une liste des revues internationales les plus importantes. 
UNESCO : Institut de statistique, OCDE : Organisation de coopération et de développement économiques, Education trends in perspective: analysis of the world education indicators, OCDE, Unesco/Paris, 2005, 228 p., stat., annexes

Ce rapport analyse l'évolution de la demande en matière d'enseignement supérieur dans dix-neuf pays émergents participant au programme WEI (World Education Indicators) entre 1995 et 2003. Il examine les facteurs qui facilitent ou freinent le développement de l'éducation dans ces pays et évalue leurs capacités à s'impliquer davantage dans la promotion des études supérieures en terme de ressources humaines et d'investissements financiers.

UNESCO : Organisation des Nations Unies pour l'éducation la science et la culturel Paris, L'enseignement supérieur dans une société mondialisée : document cadre de l'UNESCO, UNESCO/Paris, 2004, 31 pages téléchargées du site http://unesdoc. unesco.org/ [consultées en juillet 2006]

Ce document passe en revue les arguments avancés par les responsables des politiques éducatives, les chercheurs et les professionnels de l'éducation sur les opportunités et les défis auxquels la mondialisation confronte l'enseignement supérieur; recense les textes et instruments normatifs des Nations unies et de l'UNESCO pertinents pour ce débat.

\section{USHER Alex, CERVENAN Amy, Global Higher Education Rankings: Affordability} and Accessibility in Comparative Perspective 2005, Educational Policy Institute/ Toronto, 2005, 73 p. téléchargées du site http://www.educationalpolicy.org [consultation février 2006]

Cette étude réalisée par l'EPI (Educational policy institute) compare les possibilités d'accès à l'enseignement supérieur dans quinze pays : Australie, Autriche, Belgique, Canada, Finlande, France, Allemagne, Irlande, Italie, Japon, Pays-Bas, Nouvelle Zélande, Suède, Royaume-Uni, États-Unis. Des rapports nationaux comportant les résultats des enquêtes effectuées dans chaque pays figurent à la fin du document.

\section{DÉFIS ACTUELS DE L'ENSEIGNEMENT SUPÉRIEUR}

ALTBACH Philip G., Tradition and transition: the international imperative in higher education, Center for International Higher Education/Rotterdam, Sense publishers, 2007, 274 p., bibliogr.

L'enseignement supérieur est en phase de transition; encore attaché à des valeurs traditionnelles, il est en train de s'adapter aux changements induits par les nouvelles technologies, l'économie de la connaissance, la mondialisation et une forte internationalisation, tant des étudiants que des enseignants. L'auteur analyse dans une perspective comparatiste les grands défis que doit relever l'enseignement supérieur, notamment la massification de l'accès à l'enseignement supérieur dans une période de désengagement financier de l'État. Certains chapitres traitent plus particulièrement des pays en développement, des universités asiatiques, du contexte africain et des États-Unis.

Banque mondiale/Washington, Construire les sociétés du savoir : nouveaux défis pour l'enseignement supérieur, Presses de l'université de Laval/Québec, 2003, 295 p., bibliogr.

Ce rapport décrit la façon dont l'enseignement supérieur permet à un pays de prendre part à une économie mondiale de plus en plus fondée sur la connaissance. Après un chapitre sur 
l'environnement mondial et ses transformations, le rapport s'intéresse à la crise continue dont souffre l'enseignement supérieur dans les pays en développement et les pays en transition et aux relations entre les institutions de l'enseignement supérieur, les règles de marché et l'État. Il consacre un dernier chapitre aux actions menées par la Banque mondiale dans ce secteur.

\section{BOK Derek, Universities in the marketplace: the commercialisation of higher education, Princeton University Press/Oxford, 2005, 233 p.}

La marchandisation grandissante de l'enseignement supérieur s'explique par une économie fondée sur la connaissance. Dans leur souhait de générer du profit, les établissements d'enseignement supérieur risquent de négliger les valeurs de l'université et leurs missions initiales. L'auteur, ancien président de l'université de Harvard aux États-Unis, revient sur les origines de la marchandisation, ses avantages et son coût. Il réfléchit aux moyens de préserver les valeurs éducatives de l'université et de protéger l'intégrité de la recherche scientifique.

BRETON Gilles dir., LAMBERT Michel dir., DANIEL John et al., Globalisation et universités : nouvel espace, nouveaux acteurs, Presses de l'Université Laval/ Québec/Canada, UNESCO/Paris, 2003, 264 p., bibliogr.

En s'ouvrant au nouvel espace mondial, les universités voient apparaître de nouveaux acteurs tels que les régions, les entreprises, les organisations internationales ou encore les ONG; l'État national n'est plus l'unique centre autour duquel gravitent les universités. Dix-sept spécialistes de l'enseignement supérieur, venus de pays développés comme de pays en développement, s'expriment sur les enjeux que représente la globalisation pour l'enseignement supérieur. Les nouveaux acteurs sur la scène internationale, notamment les universités entrepreunariales, sont analysés.

BROCK Colin, DANIEL John, MIDDLEHURST Robin et al., First global forum on international quality assurance accreditation and the recognition of qualifications in higher education, UNESCO/Paris, 2002, 174 p.

Cet ouvrage rassemble les contributions présentées au forum universel sur la mondialisation et l'enseignement supérieur. Au-delà de l'analyse sur l'assurance qualité des enseignements et les standards internationaux d'accréditation, les auteurs proposent une réflexion sur l'enseignement supérieur : service commercial ou bien public? Cette réflexion s'organise autour des problématiques suivantes : les rapports entre l'administration d'une politique éducative et les nationalismes, la régionalisation, la mondialisation et plus particulièrement la «marchandisation» de l'éducation, enfin la diversification dans le domaine du post-secondaire, qui ouvre de nouvelles voies dans l'acquisition de compétences.

LEBEAU Yann coord., GOASTELLEC Gaële, TOMUSK Voldemar et al., Pressions sur l'enseignement supérieur au Nord et au Sud : dossier, Cahiers de la recherche sur l'éducation et les savoirs, 2006, $n^{\circ} 5,356 p$.

Des similarités existent dans les processus de réforme des systèmes d'enseignement supérieur au Nord comme au Sud et les universités semblent adopter partout des pratiques uniformes de gestion de leurs ressources humaines et matérielles. Les articles abordent l'introduction de techniques managériales dans la gestion des établissements, le découplage des fonctions d'enseignement et de recherche ou la privatisation des fonctions de services aux étudiants. Les réformes engagées tendent à produire une vision homogénéisante des systèmes nationaux d'enseignement supérieur et masquent l'extrême diversité des paysages universitaires comme le processus de fragmentation qui accompagne la réforme. 


\section{Internationalisation et mondialisation}

\section{BASHIR Sajitha, Trends in international trade in higher education: implications}

and options for developing countries, The World Bank/Washington, mars 2007, (Education. Working paper series, $n^{\circ} 6$ ), 96 pages téléchargées du site http:// siteresources.worldbank.org/ [consulté en juin 2007].

On assiste à une accélération et à une diversification des échanges internationaux de services dans l'enseignement supérieur. L'auteur en décrit les caractéristiques, notamment l'offre accrue de formation supérieure vers les pays en développement ou en transition. La quatrième partie est consacrée aux accords commerciaux sur les services et à certains accords bilatéraux.

OCDE : Organisation de coopération et de développement économiques, CERI : Centre pour la recherche et l'innovation dans l'enseignement, Enseignement supérieur : internationalisation et commerce, OCDE/Paris, 2004, 360 p.

L'enseignement postsecondaire transnational, en plein développement, se caractérise par la mobilité internationale des étudiants et par celle des programmes et des établissements éducatifs par-delà les frontières. Cet ouvrage rassemble des statistiques sur la nature et l'étendue de ce phénomène, des études de cas sur les établissements et une analyse des principales difficultés rencontrées. Il apporte une vue d'ensemble sur l'enseignement transnational et analyse son évolution en Amérique du Nord, en Europe et dans la région Asie-Pacifique. La dernière partie étudie les incidences sur l'accès et l'équité, le coût et le financement, et la qualité.

UNESCO : Institut de statistique/Montréal, Recueil de données mondiales sur l'éducation 2006 : statistiques comparées sur l'éducation dans le monde, Institut de statistique de l'Unesco/Montréal, 2006, 192 p.

Cette édition a pour objectif principal le diagnostic de l'état de l'enseignement supérieur et introduit le concept des "étudiants en mobilité internationale». Le rapport présente de nouveaux indicateurs qui tracent le flux de ces étudiants, en augmentation de $41 \%$ entre 1999 et 2004, en précisant leur destination, les impacts sur les pays d'origine et d'accueil, et les facteurs qui motivent ces étudiants. Le rapport examine également l'équilibre entre les sexes dans les différents domaines d'étude.

\section{Privatisation de l'enseignement supérieur}

ALTBACH Philip G. dir., LEVY Daniel C. dir., Private higher education: a global revolution, Sense publishers/Rotterdam, 2005, 300 p., index des auteurs (Global perspectives on higher education)

Depuis 1975, l'enseignement supérieur privé se développe plus vite que l'enseignement public, phénomène observé dans le monde entier. Cet ouvrage collectif propose d'abord une analyse globale de l'enseignement privé, notamment son rôle, les différents statuts des établissements et les politiques publiques menées. L'essentiel de l'ouvrage est consacré à des études régionales et nationales, écrites par des acteurs de l'enseignement supérieur.

DIDRIKSSON Axel coord., HERRERA Alma coord., El financiamento de la Universidad en América latina y el Carä̈be, CESU/Mexico, IESALC/UNESCO, Universidad Nacional Autonoma de México, 2006, 204 p. (Educacion superior contemporanea) 
Cet ouvrage collectif propose une analyse de la problématique du financement de l'enseignement supérieur en Amérique latine et des modalités que les «méga-universités» ont mises en place pour pallier l'absence de politique publique envers l'enseignement supérieur. Dans une première partie, on trouvera des études de cas pour le Mexique, la Colombie, le Vénézuela, Cuba, le Pérou et Costa Rica. La seconde partie présente les expériences de quatre universités mexicaines.

L'éducation : bien public et/ou marchandise? Dossier, L'Harmattan/Paris, 2006 (L'Année de la recherche en sciences de l'éducation: Année 2005), 174 p.

Les différents contributeurs analysent l'ambiguïté de l'opposition bien public/marchandise en éducation. Quatre articles portent sur l'enseignement supérieur dans les pays d'Amérique latine.

\title{
SLANTCHEVA Snejana, LEVY Daniel, Private Higher Education in Central and
} Eastern Europe: In Search of Legitimacy, Palgrave Macmillan/England, 2007, $344 p$.

Le mouvement de privatisation de l'enseignement supérieur touche les anciens pays socialistes. Dans une perspective régionale et nationale, les auteurs décrivent le rôle joué par l'enseignement privé en Europe centrale et orientale et les caractéristiques de ces nouveaux établissements, en insistant sur la question de la légitimité.

\section{VARGHESE N.V. dir., EFFAH P., AJAYI T. et al., Growth and expansion of} private higher education in Africa, IIPE/Paris, 2006, 248 p.

Depuis les années quatre-vingt-dix, l'Afrique a vu le nombre de ses établissements d'enseignement supérieur privés s'accroître, qu'il s'agisse de structures auto-financées à but lucratif ou d'établissements fondés par des organismes religieux. Généralement de petite taille, ces établissements sont axés sur des filières spécifiques en prise avec le marché. Cet ouvrage analyse les caractéristiques de l'expansion de ces établissements privés, leurs modes de gestion et leurs financements pour les pays suivants : Ghana, Kenya, Nigéria, Sénégal, Afrique du Sud, Tanzanie, Ouganda, Zimbabwe.

\section{Qualité et reconnaissance des diplômes}

\begin{abstract}
MARTIN Michaela, STELLA Antony, External quality assurance in higher education: making choices, IIPE-UNESCO/Paris, 2007, 112 p. (Fundamentals of educational planning, $n^{\circ} 85$ )

L'assurance qualité externe peut prendre des formes variées - accréditation, évaluation ou encore audit externe. Elle se fonde sur des critères également diversifiés : responsabilité financière, respect des normes, amélioration de la qualité notamment. L'ouvrage passe en revue les choix méthodologiques et organisationnels à opérer. Le dernier chapitre est consacré à l'agence chargée de cette politique d'assurance qualité.
\end{abstract}

\section{NORMAND Romuald coord., De la formation à l'emploi : des politiques à}

l'épreuve de la qualité : dossier, Éducation et sociétés, février 2006, n 18, pp. 5-109

Plusieurs articles de ce numéro portent sur les politiques de la qualité dans l'enseignement supérieur et la recherche et de leurs conséquences. L'adoption de critères de qualité tend à exacerber les tensions entre les pays du Nord et du Sud; les écarts se creusent, à l'échelon européen, entre les universités tournées vers la recherche et celles tenues de s'adapter à leur population locale. Enfin, l'évaluation de la qualité devient un gigantesque marché économique. 
OCDE : Organisation de coopération et de développement économiques, Qualité et reconnaissance des diplômes de l'enseignement supérieur : un défi international, OCDE/Paris, 2004, 228 p.

L'enseignement supérieur connaît des développements nouveaux tels que la cyberformation, les organismes de formation à caractère commercial, les campus communs et les consortiums transnationaux. Ces changements remettent en cause les systèmes nationaux d'assurance qualité ainsi que les organismes et les dispositifs d'homologation. Parallèlement, l'accroissement de la mobilité internationale des étudiants et des salariés a donné de l'importance à la reconnaissance mutuelle des diplômes et des qualifications professionnelles. Cet ouvrage dresse un panorama des moyens qu'emploient certains pays pour faire face à ces défis.

TRES Joaquim, SANYAL Bikas C., GIRO ROCA Antoni, Higher Education in the world 2007: accreditation for quality assurance: what is at stake?, Palgrave Macmillan/New York, 2007, 415 p., glossaire, bibliogr.

Ce rapport annuel du Global University Network for Innovation (GUNI) rassemble cette année les questions autour de la qualité de l'enseignement supérieur au vingt-et-unième siècle. En se basant sur des analyses théoriques et des expériences pratiques, les contributeurs s'expriment, au niveau mondial et régional, sur le développement de la qualité dans l'enseignement supérieur, sur l'arrière plan historique et sociologique, sur ses mécanismes, les implications financières qu'elle comporte, le contrôle de la corruption et les différentes étapes à mettre en œuvre.

\section{Les acteurs de l'enseignement supérieur}

COALDRAKE Peter, DUNKIN Ruth, CONLON Michael et al., Répondre aux attentes des étudiants, OCDE/Paris, 2002, 168 p., bibliogr., schémas, données chiffrées

Le Programme sur la gestion des établissements d'enseignement supérieur de l'OCDE a défini un projet visant à informer les établissements de l'évolution des attentes des étudiants. En effet, les universités doivent s'adapter à la diminution sensible de leurs ressources et aux exigences d'un public de plus en plus diversifié. Ce volume, reprenant des communications de séminaires organisés en 2001, analyse la nature des rapports entre étudiants et universités et les perspectives de l'action des pouvoirs publics.

\section{HADJI Charles, BARGEL Tino, MASJUAN Josep, Étudier dans une université qui change : le regard des étudiants de trois régions d'Europe, PUG/Grenoble/ France, 2005, 285 p. \\ Cet ouvrage présente les résultats d'une enquête conduite en 2001-2002 dans trois régions d'Europe : le Bade-Wurtemberg, la Catalogne et la région Rhône-Alpes. L'ouvrage se struc- ture autour de questions sur les motivations des étudiants, leurs attentes, leur vie d'étudiant, les comportements d'apprentissage et de travail ou leurs représentations de l'avenir. Une première partie présente le jugement des étudiants sur leurs études et les enseignements qu'ils reçoivent. L'inégalité sociale entre les étudiants et les effets du genre sur leurs comportements et attentes est ensuite analysée suivie de la professionnalisation de l'université.}

MUSSELIN Christine, Le marché des universitaires. France, Allemagne, ÉtatsUnis, Presses de Sciences Po/Paris, 2005, 325 p. (Collection Académique)

La gestion des carrières universitaires, enjeu majeur pour l'autonomie des universités, occupe une place centrale dans les réformes de l'enseignement supérieur. L'auteure s'est appuyée sur 
une enquête pour comparer les pratiques de recrutement des universitaires dans trois pays aux traditions très différentes : l'Allemagne, les États-Unis et la France. Ce livre débouche sur une analyse inédite des marchés du travail universitaires et ouvre la discussion sur les réformes à engager et la manière de les conduire.

\section{REGE COLET Nicole, ROMAINVILLE Marc, La pratique enseignante en} mutation à l'université, De Boeck Université/Bruxelles/Belgique, 2006, 249 p. (Perspectives en éducation et formation)

Les universités sont souvent accusées de négliger leur mission d'enseignement au profit de la course à l'excellence dans la recherche. L'objectif de cet ouvrage est de faire le point sur les mutations pédagogiques en cours dans l'enseignement universitaire. La première partie est consacrée à un diagnostic des malaises vécus par l'université sur ses missions d'enseignement au travers du prisme de l'évaluation. Dans la deuxième partie, sont rassemblés des comptes rendus de pratiques pédagogiques innovantes. La dernière partie regroupe des présentations de programmes particuliers de soutien et d'accompagnement.

\section{Recherche}

\section{CONNELL Helen dir., La gestion de la recherche universitaire : relever le défi au niveau des établissements, OCDE/Paris, 2004, 293 p.}

L'efficacité de la gestion de la recherche devient une question décisive pour les établissements d'enseignement supérieur et la production scientifique d'un établissement est cruciale pour sa compétitivité et son prestige dans le monde. Ce rapport s'articule autour de quatre thèmes : importance grandissante de la mission de recherche pour l'enseignement supérieur, renforcement des structures et des méthodes de gestion, ressources financières affectées et développement des carrières de chercheur. On y trouve également huit études de cas en Allemagne, en Australie, en Belgique, au Brésil, en Irlande, en Malaisie, au Portugal et en Turquie.

Institut Montaigne, Avoir des leaders dans la compétition universitaire mondiale, Institut Montaigne/Paris, octobre 2006, 116 p. téléchargées du site http://www. institutmontaigne.org [consultation octobre 2006]

L'Institut Montaigne, laboratoire d'idées (think tank), a pour objectif d'identifier les critères essentiels permettant de créer en France des établissements d'enseignement supérieur d'excellence. Ce rapport décrit les enjeux des universités de recherche et des établissements d'enseignement supérieur et de recherche directement exposés à la concurrence internationale. Enfin, il définit les critères qui permettraient à la France de posséder des établissements susceptibles de rivaliser avec les meilleures écoles mondiales. Le rapport présente deux exemples étrangers : le Massachussets Institute of Technology (MIT) et l'École polytechnique fédérale de Lausanne.

\section{HAZELKORN Ellen, La gestion de la recherche universitaire : développer la recherche dans les nouveaux établissements, OCDE/Paris, 2005, 243 p., annexes}

Cette étude internationale, à laquelle vingt-cinq établissements d'enseignement supérieur (ESS) de dix-sept pays ont pris part, analyse les procédures et les stratégies élaborées par les nouveaux établissements d'enseignement supérieur pour développer leur activité de recherche. À la lumière des études de cas, des recommandations sont faites à l'intention des dirigeants d'établissements et des pouvoirs publics. 


\title{
ÉTUDES TRANSNATIONALES ET NATIONALES
}

\begin{abstract}
Afrique
AFFA'A Félix-Marie, DES LIERRES Thérèse, L'Afrique noire face à sa laborieuse appropriation de l'université : les cas du Sénégal et du Cameroun, L'Harmattan/ Paris, Presses de l'Université Laval/Québec/Canada, 2002, 321 p.

Après une présentation générale de l'université en Afrique, l'ouvrage propose une analyse historique et sociologique du curriculum universitaire de deux universités, l'université de Dakar et l'université de Yaoundé, et identifient les raisons des échecs des réformes qui y ont été entreprises.
\end{abstract}

AKAM Noble dir., DUCASSE Roland dir., Quelle université pour l'Afrique?, Maison des sciences de l'homme d'Aquitaine/Pessac, 2002, 314 p. (Dynamique locale de la mondialisation, les Afriques en perspective)

Les universités africaines sont actuellement dans une situation de crise; les conséquences et les efforts pour en sortir sont l'objet d'analyses dont l'ouvrage rend compte. Il est constitué de cinq parties : carrières universitaires; diplômes et insertion professionnelle; campus entre crises et réformes; nouveaux outils de formation : campus virtuels; nouvelles formes d'organisation.

GIOAN Pierre Antoine, Enseignement supérieur en Afrique francophone : quels leviers pour des politiques financièrement soutenables?, World Bank/Washington, mars 2007, 37 pages téléchargées du site http://siteresources.worldbank.org/ EDUCATION (Série : le développement humain en Afrique) [consulté le 10 mai 2007]

Depuis plus d'une décennie, l'enseignement supérieur et la recherche (ESR) dans les pays francophones d'Afrique sont confrontés à une crise profonde dont l'origine se situe principalement dans le déséquilibre croissant entre les besoins nécessaires pour assurer un enseignement de qualité et les ressources disponibles. Cette publication a pour objectif d'en déterminer les facteurs communs, d'identifier les conditions permettant d'inscrire les dispositifs d'ESR dans des évolutions maîtrisées financièrement et de proposer des pistes pour améliorer le financement de ce secteur et préserver sa qualité.

\section{Amériques}

ALTBACH Philip G. ed., BERDAHL Robert O. ed., GUMPORT Patricia J. ed, American higher education in the twenty-first century: social political and economic challenges, Johns Hopkins university press/Baltimore/États-Unis, 2005, 558 p., bibliogr., index thématique

Les défis que doit relever l'enseignement supérieur américain en ce début de $\mathrm{XXI}^{\mathrm{e}}$ siècle sont d'ordre politique, social et économique. Après un panorama historique et une comparaison avec d'autres pays, les thématiques de l'autonomie, de la responsabilité financière, et de la liberté académique y sont analysées : le financement, la technologie, les diplômes, les curricula et les marchés qui sous-tendent l'enseignement supérieur.

Conseil supérieur de l'Éducation/Québec, L'internationalisation : nourrir le dynamisme des universités québécoises, Conseil supérieur de l'éducation/Sainte-Foy, novembre 2005, 104 p. 
Dans cet Avis, le Conseil décrit le développement des activités internationales des universités québécoises et en dégage les orientations en matière d'internationalisation depuis les années 1970.

\title{
OCDE, L'enseignement supérieur en Amérique latine : la dimension inter- nationale OCDE/Paris, 2005, 397 p.
}

Cette publication présente une analyse comparative des problèmes, tendances et possibilités liées à l'internationalisation de l'enseignement supérieur, analyse conduite dans sept pays d'Amérique latine (Argentine, Brésil, Chili, Colombie, Cuba, Mexique et Pérou). Cet examen passe par l'évaluation du développement de l'enseignement supérieur en Amérique latine selon plusieurs facteurs : cursus, réseaux éducatifs, mobilité des enseignants et étudiants.

\begin{abstract}
Asie
HARAYAMA Yuko, Le système universitaire japonais, Economica/Paris, 2000, 124 p. (Université)

L'université japonaise fait face à des phénomènes nouveaux : la population de jeunes de dix-huit ans décroît et le secteur productif réclame des diplômés créatifs et hautement spécialisés. Cette étude s'intéresse plus particulièrement à l'université de recherche. L'auteur analyse l'état de la question sur la formation supérieure en Europe et aux États-Unis, l'évolution de la structure du système universitaire japonais, l'enseignement au sein de l'université, la politique universitaire au Japon puis l'université en tant qu'organisme de recherche.
\end{abstract}

\section{MEEK Lynn, SUWANWELA Charas, Higher education, research and knowledge} in the Asia-Pacific region, Palgrave Macmillan/Grande-Bretagne, 2007, 256 p. (Issues in higher education)

Axé sur la région Asie-Pacifique, cet ouvrage dresse un état de lieux de la production de connaissance et de la recherche au sein des établissements d'enseignement supérieur. Dans une perspective comparatiste et critique, il analyse si les pays concernés offrent un environnement favorable ou non à la promotion de la recherche au sein de l'enseignement supérieur.

OCDE : Organisation de coopération et de développement économiques/Paris, Current issues in Chinese higher education, OCDE/Paris, 2000, 144 p. (Education and skills)

Cet ouvrage reprend les interventions du séminaire consacré aux nouveaux modes de gestion universitaire en Chine. Les thématiques ont porté sur la liberté de l'enseignement et ses obligations, l'autonomie des universités, le choix du meilleur modèle de réforme du système administratif chinois, la diversification des sources de financement pour les universités, les coûts en personnel, l'innovation, la coopération souhaitée entre la recherche universitaire et l'industrie au service de l'économie nationale, enfin l'internationalisation.

\section{Afrique du Nord et Proche-Orient}

BASHSHUR Mounir dir., COURBAGE Youssef dir., LABAKI Boutros dir., L'enseignement supérieur dans le monde arabe : une question de niveau?, Institut français du Proche-Orient/Liban, 2006, 214 p.

Cet ouvrage (en arabe, français et anglais) fait suite à un atelier international organisé en 2005 à Beyrouth. Les auteurs observent l'organisation universitaire de six pays d'Afrique du Nord et du Proche-Orient au regard de leurs contraintes démographique et financière, et dressent 
un état des lieux du niveau et de la qualité de l'enseignement dans le monde arabe. Un dernier chapitre offre une vision comparatiste sur la base des différentes analyses et des discussions tenues durant l'atelier international.

CPU/Conférence des Présidents de l'Université, AUF/Agence Universitaire de la Francophonie, Les réformes de l'enseignement supérieur des pays du Maghreb et la perspective du processus de Bologne : actes du colloque-Marseille 19 et 20 novembre 2004, CPU/Paris, 2006, 92 pages téléchargées du site http://www. cpu.fr/ [consulté en février 2006]

Cette rencontre des recteurs et présidents d'universités des pays du Maghreb et des Conférences francophones de l'Union européenne a donné lieu à des actes. On y trouve la présentation des réformes en cours et des priorités pour les universités du Maghreb et celles des pays européens, les outils institutionnels du partenariat entre établissements. Une dernière partie énonce des propositions pour une coopération euro-méditerranéenne.

\section{Europe}

DEER Cécile, Higher Education in England and France since the 1980s, Symposium Books/Oxford, 2002, 208 p., bibliogr.

Cet ouvrage propose une analyse synthétique du développement de l'enseignement supérieur en Grande-Bretagne et en France. L'objectif est d'identifier les points de divergence et les similitudes concernant la politique, les idéologies sous jacentes et l'organisation des systèmes d'enseignement supérieur de chacun des deux pays.

EURYDICE : Réseau d'information sur l'éducation en Europe/Bruxelles, Focus sur les structures de l'enseignement supérieur en Europe 2004/2005. Évolutions nationales dans le cadre du Processus de Bologne, Eurydice/Bruxelles, 2005, 234 p. Cette publication présente les structures de l'enseignement supérieur en Europe à travers des diagrammes détaillés. Les principaux parcours de formation pour l'année 2004-2005, les conditions d'accès aux filières et leurs durées respectives sont présentés pour les quarante pays actuellement signataires de la Déclaration de Bologne. Chaque diagramme est complété par un texte synthétique qui dresse un état des lieux des réformes ou dispositifs introduits en lien avec le Processus de Bologne, depuis 1999. En introduction, le rapport présente un bref historique du processus de Bologne.

EURYDICE : Réseau d'information sur l'éducation en Europe/Bruxelles, Focus on Higher education in Europe 2006/2007. National trends in the Bologna process, Eurydice/Bruxelles, 2007, 349 p.

Cette publication présente l'évolution de l'organisation de l'enseignement supérieur des quarante-cinq pays signataires de la Déclaration de Bologne au moment de la Conférence de Londres (mai 2007). Elle propose un état des lieux de chacun des objectifs du processus : adoption d'une structure en trois cycles (licence, mastère, doctorat-LMD), introduction du système européen de transfert et d'accumulation de crédits (ECTS), délivrance du supplément au diplôme, reconnaissance des diplômes conjoints, mise en place de cadres nationaux de certifications et développement de l'assurance qualité.

ELLIOT Imelda, Irlande : l'enseignement supérieur en mutation 1960-1997, L'Harmattan/Paris, 2006, 257 p., bibliogr.

L'auteure présente un panorama global de l'évolution du système d'enseignement supérieur en République d'Irlande selon trois angles d'approche : l'évolution des établissements, celle de l'administration du système et celle du recrutement des étudiants. 
HALIMI Suzy, L'enseignement supérieur au Royaume-Uni, Ophrys/Paris, 2004, 176 p., bibliogr. (Civilisation)

L'ouvrage offre un tableau de l'enseignement supérieur au Royaume-Uni dans une dimension historique et prospective. Pour comprendre la situation actuelle, l'auteure retrace l'histoire de l'enseignement supérieur depuis le Moyen Âge jusqu'au XX ${ }^{e}$ siècle avec les missions qui sont assignées à l'université : équité (plus grande démocratisation) et efficacité (participer à l'expansion économique). La seconde partie définit les enjeux pour le $\mathrm{XXI}^{\mathrm{e}}$ siècle : structures, financement de l'enseignement supérieur, accès, taux de réussite ou insertion professionnelle des diplômés.

OCDE : Organisation de coopération et de développement économiques, L'enseignement tertiaire en Suisse, OCDE/Paris, 2003, 238 p. (Examens des politiques nationales d'éducation)

Ce document est constitué d'un rapport de base établi par les autorités suisses suivi de celui des examinateurs de l'OCDE. Dans sa première partie, les auteurs situent le contexte dans lequel s'inscrit le degré tertiaire de l'éducation avec un descriptif du pays et du système éducatif, les défis et les diverses réformes en cours. Les examinateurs de l'OCDE développent des analyses et proposent des recommandations pour l'amélioration du système d'enseignement supérieur suisse.

OCDE : Organisation de coopération et de développement économiques, L'enseignement supérieur en Irlande, OCDE/Paris, 2006, 297 p. (Examens des politiques nationales d'éducation)

Ce rapport est le résultat d'une enquête menée par l'OCDE sur l'enseignement supérieur en Irlande. Les examinateurs analysent les principaux défis du système d'enseignement supérieur et recommandent une série d'actions. Les autorités irlandaises présentent ensuite le système et la politique d'éducation, l'objectif déclaré étant de "placer son système d'enseignement supérieur dans le groupe de tête des pays de l'OCDE en termes de qualité et de niveau de participation».

\section{Harmonisation de l'espace européen en matière d'enseignement supérieur}

\section{HERBILLON Michel, Rapport d'information sur l'enseignement supérieur en Europe, Assemblée nationale/Paris, 2004, 130 p.}

Après avoir exposé les raisons pour lesquelles le Processus de Bologne est non seulement un objectif pour l'Europe mais aussi une chance pour la France, l'auteur présente les points forts de l'attractivité des universités américaines et insiste sur l'urgence d'une réponse européenne face aux États-Unis. Enfin, douze propositions sont faites concernant l'enseignement supérieur français en Europe et l'enseignement supérieur européen dans le monde.

GERARD Patrick dir., VANDENBERGHE Vincent dir., L'enseignement supérieur après Bologne, Reflets et Perspectives de la vie économique, 2006, vol. $n^{\circ} 2, n^{\circ} 45$, $108 \mathrm{p}$.

Plusieurs articles de ce numéro portent sur le financement de l'enseignement supérieur dans différents contextes, sur la mobilité et la recherche avec la mise en place de l'espace européen de l'enseignement supérieur.

LAVDAS Kostas A., PAPADAKIS Nikos E., GIDARAKOU Marrianna, Construction de l'espace européen de l'enseignement supérieur : les grandes orientations et les réseaux, Politiques et gestion de l'enseignement supérieur, janvier 2006, vol. $n^{\circ} 18, n^{\circ} 1$, pp. 145-155, bibliogr. 


\section{FRANCE}

Les étudiants in Repères et références statistiques sur les enseignements la formation et la recherche : 2006, Ministère de l'éducation nationale. DEPP/ Direction de l'évaluation de la prospective et de la performance, 2006, pp. 169-211 (RERS)

Série de données statistiques sur les effectifs d'étudiants en France par académie, filière, universités, IUT, écoles de commerce. On y trouve également des données sur l'origine socioprofessionnelle des étudiants, les flux, les parcours et l'évolution des taux d'inscription.

\section{MALLET Daniel, BALME Pierre, RICHARD Pierre et al., Réglementation et management des universités françaises, PUG/Grenoble/France, 2005, 605 p., (Libres cours)}

Cet ouvrage traite des questions soulevées par la gestion des universités au travers de témoignages de praticiens et responsables actuels. Après une partie sur l'organisation et les règles de fonctionnement des universités, il examine leurs missions (formation, recherche, vie étudiante), leurs moyens; enfin, il aborde une nouvelle discipline, le management universitaire (acteurs, partenaires, modes de management).

REY Olivier, L'enseignement supérieur sous le regard des chercheurs, Veille scientifique et technique, mars 2005, téléchargé sur le site de l'INRP http://www. inrp.fr/ [consultation mai 2007], 53 p.

Ce dossier, réalisé en partenariat avec le Réseau d'étude sur l'enseignement supérieur, vise à restituer les axes principaux des recherches menées en France sur l'enseignement supérieur qui se sont fortement développés depuis une dizaine d'années. Une bibliographie générale et une sitographie (sites français, sélection de sites et de revues internationales du domaine) complète l'ensemble.

\section{Rapports officiels}

ADNOT Philippe, La valorisation de la recherche dans les universités, Sénat/ Paris, 2006, $n^{\circ} 341,79$ pages téléchargées de http://www.senat.fr [consultées en juillet 2006]

Ce rapport du Sénat est le résultat d'une mission de contrôle sur la valorisation de la recherche dans les universités françaises. Elle s'est faite à partir des résultats d'un questionnaire envoyé à l'ensemble des universités françaises, d'auditions de plusieurs responsables de valorisation et d'entretiens dans des pays ayant développé des politiques volontaristes en ce domaine (Israël, Québec, Finlande).

AGHION Philippe, COHEN Elie, Éducation et croissance, Paris : Documentation française, 2004, 143 p.

Le rapport dénonce une organisation de l'enseignement supérieur marquée par une coupure entre les grandes écoles dotées de moyens financiers importants et les universités, parents pauvres du système. La massification et la démocratisation des universités donnent nombre d'échecs en DEUG et les universités pâtissent d'une faible sélection. Le rapport présente par ailleurs les spécificités et l'évolution du modèle universitaire français et propose trois scénarios d'évolution de l'université en précisant les principaux leviers de la réforme. 
ATTALI Jacques dir., Pour un modèle européen d'enseignement supérieur : rapport au Ministre de l'Éducation nationale, de la recherche et de la technologie, Paris : Ministère de l'éducation nationale, 1998, 77 pages téléchargées du site http://www.education.gouv.fr/

Ce rapport est le résultat d'une consultation d'universitaires, de chercheurs et de chefs d'entreprise, sur le système d'enseignement supérieur français et son intégration dans l'Union européenne. Il analyse la situation de l'enseignement supérieur français (filières, apprentissage des savoirs, relations avec l'État et les entreprises) et les réformes nécessaires des universités et grandes écoles. Des synthèses des réformes en cours dans d'autres pays (Allemagne, Italie, Royaume-Uni, États-Unis) sont présentées.

CNE : Comité national d'évaluation des établissements publics à caractère scientifique culturel et professionnel/Paris, Nouveaux espaces pour l'université : Rapport au Président de la République 2000-2004, Documentation française/ Paris, 2005, 208 p.

Ce rapport a pour objet de dresser un bilan de synthèse sur l'état de l'enseignement supérieur en France. La première partie rend compte des évaluations réalisées durant les années 2000-2004. Le CNE a engagé son action dans deux directions : une nouvelle méthodologie d'évaluation et le développement des coopérations dans le cadre de la construction de l'espace européen de l'enseignement supérieur. L'ancrage territorial des universités et le positionnement de l'offre de formation universitaire dans le "post-bac» font également l'objet d'analyses. Le rapport se clôt sur le point de vue du CNE dans les débats sur la modernisation des universités et l'harmonisation européenne.

DEJEAN Jacques, L'évaluation de l'enseignement dans les universités françaises, Haut conseil de l'évaluation de l'école/Paris, 2002, 108 p.

Ce rapport cherche à établir un état des lieux de l'évaluation de l'enseignement dans les universités françaises quatre ans après l'arrêté du 9 avril 1997 invitant les universités à organiser une procédure d'évaluation des enseignements et des formations. Le rapport porte sur l'enseignement tel qu'il est conçu (définition des objectifs, des contenus, des méthodes, des pratiques d'évaluation) et tel qu'il est réalisé par les enseignants.

GOULARD François dir., L'enseignement supérieur en France : état des lieux et propositions, Ministère de l'Éducation Nationale de l'enseignement supérieur et de la Recherche/Paris, 2007, 150 p. téléchargées sur le site http://www.recherche.gouv.fr [consulté en mai 2007]

Ce rapport établit un état des lieux critique et prospectif de l'enseignement supérieur français. Il présente l'offre française de formation supérieure dans toutes ses composantes ainsi que l'employabilité et l'insertion des diplômés suivie d'une analyse de la diversité des filières de formation. La dernière partie propose une réforme de la gouvernance des universités, quelques pistes d'action sur l'enseignement professionnel supérieur, le cycle de la licence et la formation continue.

\footnotetext{
HARFI Mohamed, MATHIEU Claude, Étudiants et chercheurs à l'horizon 2020 : enjeux de la mobilité internationale et de l'attractivité de la France, Documentation française/Paris, septembre 2005, 249 p.

Ce rapport, élaboré par le Commissariat général du Plan, analyse les enjeux de la mobilité internationale et les différentes stratégies pouvant favoriser l'attractivité de la France. Elle est essentielle à l'amélioration de la compétitivité et au dynamisme des systèmes d'enseignement supérieur et de recherche. Cinq scénarios à l'horizon 2020 sont élaborés et comparés au regard des objectifs stratégiques de mobilité internationale et d'attractivité de la France.
} 


\section{Haut Comité éducation économie/Paris, Objectif $50 \%$ d'une génération diplômée de l'enseignement supérieur, Documentation française/Paris, 2006, 256 p.}

L'accès au baccalauréat et à l'enseignement supérieur a fortement progressé dans les années quatre-vingt, puis s'est stabilisé autour de 1995. Avec 42 diplômés du supérieur pour 100 jeunes, la France se situe dans la moyenne des pays de l'OCDE. L'objectif de $50 \%$ de diplômés est-il conforme aux attentes de la société? Ce rapport énonce des préconisations pour une meilleure articulation entre enseignement secondaire et supérieur, la diminution des sorties sans diplôme et l'amélioration de l'insertion dans le monde du travail. Le rapport, réalisé pour le HCEEE, porte sur les travaux de recherche à l'appui du débat.

HETZEL Patrick, BARET Laurence, BEVALOT Françoise et al., De l'université à l'emploi, Commission du débat national sur l'avenir de l'Ecole/Paris, octobre 2006, 108 p. téléchargées $d u$ site http://www.debat-universite-emploi.education.fr, bibliogr. [consultation octobre 2006]

Comment réduire les taux d'échec dans certaines filières universitaires et améliorer l'insertion professionnelle des étudiants? Ce rapport propose de redéfinir les procédures d'orientation pré et post-bac et d'améliorer la dimension professionnelle des formations universitaires par le rapprochement durable de l'université et du marché de l'emploi.

\section{Mutations de l'enseignement supérieur français}

CYTERMANN Jean-Richard, Universités et grandes écoles : dossier, Problèmes politiques et sociaux, $n^{\circ}$ 936, mai 2007, $117 p$.

L'enseignement supérieur français se caractérise par la dualité universités-grandes écoles. Les universités sont concurrencées par les grandes écoles pour la formation des élites et par les organismes de recherche dans le développement de la recherche publique. Un tel système est-il performant et attractif dans la compétition internationale actuelle? Les textes présentés analysent les conséquences de cette dualité en termes de démocratisation de l'enseignement supérieur et de performance et examinent les perspectives de rapprochement entre universités et grandes écoles.

\section{DURU-BELLAT Marie, L'inflation scolaire : les désillusions de la méritocratie,} Seuil/Paris, 2006, 106 p. (La République des idées)

L'auteure met en lumière l'absence de corrélation nette, à l'échelle nationale ou internationale, entre les progrès de la scolarisation de masse des cinquante dernières années et le taux de mobilité sociale, et tente de répondre à différentes interrogations : quels bénéfices la société tire-t-elle d'une population de plus en plus instruite? Faut-il continuer à allonger les scolarités? Enfin, elle propose une réflexion sur des modalités alternatives de la sélection scolaire et sociale.

FELOUZIS Georges, La condition étudiante : sociologie des étudiants et de l'université, PUF/Paris, 2001, 299 p. (Sociologie d'aujourd'hui)

Le sentiment partagé par la plupart des étudiants en premier cycle est d'être seul et "confronté à une institution faible dont les modes de régulation ont disparu». Cet ouvrage, fruit d'une enquête de deux ans auprès d'étudiants en premier cycle (1996-1998), en fait la démonstration. La première partie analyse les données et traite de la "condition étudiante». Il aborde ensuite la dispersion du système universitaire en unités de formation de plus en plus autonomes. La question de nouveaux modèles pédagogiques et de leur mise en œuvre est posée dans une dernière partie. 
FELOUZIS Georges dir., Les mutations actuelles de l'université, Presses Universitaires de France, 2003, 400 p.

Les contributions présentées lors d'un colloque sur l'enseignement supérieur (Bordeaux, 2002) analysent les mutations de l'université. Les sujets abordés traitent des politiques de régionalisation des universités et des relations que celles-ci entretiennent avec l'industrie, de la mise en perspective du cas français par l'analyse des politiques universitaires dans quatre pays et $\mathrm{du}$ fonctionnement des établissements tant du point de vue du recrutement que de la politique de recherche.

MUSSELIN Christine, La longue marche des universités françaises, PUF/Paris, 2001, 218 p., bibliogr. (Sciences sociales et sociétés)

L'auteure décrit les caractéristiques de l'enseignement universitaire français avant les changements intervenus à la fin des années quatre-vingts et analyse les transformations produites ces dix dernières années : introduction de formations non traditionnelles, politique de contractualisation et transformation des modes de pilotage. Enfin, elle propose un cadre d'analyse des systèmes universitaires et de leurs évolutions.

ORIVEL François, Pourquoi les universités françaises sont-elles si mal classées dans les palmarès internationaux?, Les Notes de l'IREDU, mai 2004, $n^{\circ} 04 / 04$, 4 pages téléchargées du site http://www.u-bourgogne.fr [consultées en juin 2007]

\section{PERETTI Claudine dir., L'enseignement supérieur : grandes évolutions depuis} 15 ans, Éducation et formations, mars 2004, $n^{\circ} 67,154 p$.

Le numéro porte sur l'évolution des effectifs au cours de la décennie 90 avec la modification des flux entre les filières, les rééquilibrages géographiques et la part prépondérante des femmes dans l'accroissement des effectifs. Il présente un bilan de l'évolution des emplois d'enseignantchercheur suivi de contributions sur les évolutions de l'enseignement supérieur dans deux régions de France et en Allemagne. Enfin, trois études sont consacrées à l'articulation entre la formation et l'emploi.

VASCONCELLOS Maria, L'enseignement supérieur en France, La Découverte/ Paris, 2006, 121 p. (Repères)

L'ouvrage présente l'enseignement supérieur français et ses grands regroupements institutionnels avec d'un côté les grandes écoles et les écoles professionnelles relevant de filières sélectives et, de l'autre, les universités composées essentiellement de filières ouvertes à tous les bacheliers. Les derniers chapitres sont consacrés aux filières des universités dont l'offre de formation s'est transformée profondément sous la pression du nombre et des nouvelles attentes des étudiants. 